\title{
Efficacy of Navigation in Skull Base Surgery Using Composite Computer Graphics of Magnetic Resonance and Computed Tomography Images
}

\author{
Nakamasa HAYASHI, Masanori KURIMOTO, Yutaka HiRASHIMA, Hiroaki IKEDA, \\ Takashi SHiBATA, Takahiro TOMITA, and Shunro ENDO
}

Department of Neurosurgery, Toyama Medical and Pharmaceutical University, Toyama

\begin{abstract}
The efficacy of a neurosurgical navigation system using three-dimensional composite computer graphics (CGs) of magnetic resonance (MR) and computed tomography (CT) images was evaluated in skull base surgery. Three-point transformation was used for integration of MR and CT images. MR and CT image data were obtained with three skin markers placed on the patient's scalp. Volume-rendering manipulations of the data produced three-dimensional CGs of the scalp, brain, and lesions from the MR images, and the scalp and skull from the CT. Composite CGs of the scalp, skull, brain, and lesion were created by registering the three markers on the three-dimensional rendered scalp images obtained from MR imaging and CT in the system. This system was used for 14 patients with skull base lesions. Threepoint transformation using three-dimensional CGs was easily performed for multimodal registration. Simulation of surgical procedures on composite CGs aided in comprehension of the skull base anatomy and selection of the optimal approaches. Intraoperative navigation aided in determination of actual spatial position in the skull base and the optimal trajectory to the tumor during surgical procedures.
\end{abstract}

Key words: skull base surgery, transsphenoidal surgery, navigation, simulation

\section{Introduction}

Safe resection of lesions in the anatomically complex region of the skull base ultimately depends on the skill and experience of the surgeon, but intraoperative guidance systems utilizing mechanical articulated arms, free-floating probes, or navigational microscope can be useful in skull base surgery. ${ }^{1,2,4-6,8)}$ Neither brain nor soft tissue shifts reduce the accuracy of the navigation system in the skull base area. A navigation system is helpful even for large cranial base lesions. ${ }^{5}$ The use of a navigation system was evaluated in a series of 325 cases, finding that the navigation system was most useful during resection of skull base lesions. ${ }^{4)}$

We have developed a neurosurgical navigation system using three-dimensional composite computer graphics (CGs) derived from magnetic resonance (MR) and computed tomography (CT) images. ${ }^{7)}$ These three-dimensional composite CGs of the scalp, brain, skull, and lesions allow preoperative simulation of the surgical procedures and in-

Received July 25, 2000; Accepted April 18, 2001 traoperative navigation. This system was used during open brain surgery for 85 patients, including 14 patients with skull base lesion.

\section{Materials and Methods}

\section{System design}

The system (EVANS; Tomiki Medical Instrument Co., Ltd., Kanazawa) was reported in detail previously. ${ }^{7)}$ Briefly, the arm-based frameless stereotactic system provides real-time anatomical and position information to the surgeon. The articulated arm has six degrees of freedom and carries an interchangeable probe shaped like a bayonet on its tip (Fig. 1). The axis of the terminal joint is aligned with the direction from the holder of the arm to the probe tip, so no rotation of this joint can alter the tip position. Therefore, the surgeon can observe the actual spatial position of the tip and direction of the probe in relation to the images of the patient. The bayonet probe can be passed through narrow openings and does not obstruct the view of deep structures during microscopic neurosurgical procedures. Furthermore, the arm is provided with a counterbalancing 




Fig. 1 Photograph of the articulated arm with six degrees of freedom and an interchangeable bayonet probe.

weight, so the surgeon can easily hold and manipulate the probe in one hand and surgical instruments in the other hand.

\section{Imaging protocol}

Imaging studies were performed a few days before the surgical procedure. Fiducial markers were applied to the scalp using 3-mm cotton balls soaked in oil, or recently clofibrate capsules, for MR imaging, and 3-mm acrylic balls for CT. At least five markers were placed on region surrounding the surgical field. These sites were labeled with India ink for use in image registration at surgery. In addition, three markers were placed on the nasion and in the meatus of the bilateral ears to determine reference coordinates for the MR and CT images. MR imaging was performed with the following parameters for $T_{1}$ weighted images: volumetric gradient echo pulse sequence; flip angle, 40 degrees; repetition time, $40 \mathrm{msec}$; echo time, $5 \mathrm{msec} ; 256 \times 256$ image matrix; field of view, $270 \mathrm{~mm}$; and 120 contiguous sagittal slices of $1.5 \mathrm{~mm}$ thickness. The imaging protocol for CT used 2-mm thick slices with table increments of $3 \mathrm{~mm}$.

\section{Image integration}

The MR and CT image data were entered in the graphic workstation and subjected to volume-
Table 1 Clinical entities

\begin{tabular}{lc}
\hline \multicolumn{1}{c}{ Disease } & No. of cases \\
\hline Pituitary adenoma & 5 \\
Meningioma & 2 \\
Neurinoma & 2 \\
Epidermoid cyst & 2 \\
Rathke's cleft cyst & 1 \\
Chordoma & 1 \\
Hemifacial spasm & 1 \\
\hline
\end{tabular}

rendering and surface-rendering manipulations to produce three-dimensional CGs of the skin, brain, and lesions from the MR images, and of the skin and skull from the CT images. Three-point transformation was used for integration of the MR and CT images. Spatial matching of the MR and CT images used the three fiduciary points at the nasion and in the meatus of the bilateral ears on the three-dimensional CGs of the skin reconstructed from the MR and CT images. This process easily provided composite three-dimensional CGs of the MR and CT images, which were used for preoperative simulation of the surgical procedures and intraoperative navigation of the scalp, brain, lesion, and skull.

\section{Results}

This system was used for 14 patients with skull base lesions (Table 1). The bayonet probe could easily be placed in narrow surgical fields without obstructing the microscopic view of deep structures. Six transsphenoidal procedures were performed on four cases of pituitary adenoma, one of Rathke's cleft cyst, and one of clival chordoma, including two reoperations and one for poor pneumatization of the sphenoid sinus.

The three-dimensional composite CGs were easily be created using the three-point transformation process. No distortion of the MR images was recognized with the imaging parameters used in this system, and there was no effect on the accuracy of the spatial matching of MR and CT images. The threedimensional CGs of the scalp reconstructed from MR and CT images required only 5 minutes to create using the three-point transformation process. Preoperative reconstruction of the three-dimensional composite CGs required about 1 hour, including data transfer and segmentation of the brain, vessels, lesion, and skull. The composite CGs were useful for understanding the skull base anatomy.

Figure 2 shows a working display and a magnified intraoperative view via the anterior transpetrosal approach in a patient with trigeminal neurinoma. 


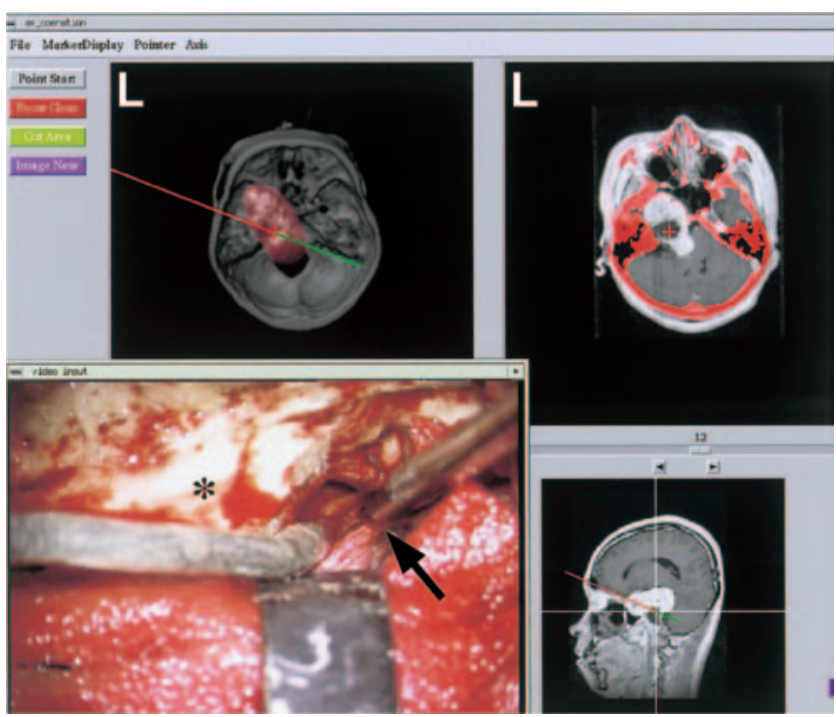

Fig. 2 Working display and magnified intraoperative view in a patient with trigeminal neurinoma. The position of the probe tip (crosshairs) and the trajectory (red and green lines) are monitored on the three-dimensional composite color graphics of magnetic resonance and computed tomography images (upper left) and also on the three reformed images (right). Asterisk indicates the petrous bone and the arrow indicates the probe tip in the magnified intraoperative view (lower left).

The probe tip could easily be placed in the narrow surgical field and pointed at the tumor. The position of the probe tip was monitored on the three-dimensional composite CGs and on the three reformed planes (axial, coronal, and sagittal) of the MR and CT images as well. Our system provided useful information concerning expansion of the tumor in the skull base and optimal approaches, and was helpful in determining the adequate extent of resection of the petrous bone and the optimal trajectory to the tumor during the surgical procedures.

Figure 3 shows a working display and a magnified intraoperative view via the transsphenoidal approach in a patient with pituitary adenoma. The position of the probe tip was monitored on the triorthogonal reconstruction (Fig. 3A) and the threedimensional composite CGs of the MR and CT images (Fig. 3C). We could safely confirm the appropriate trajectory to the sella and locate the midline. The system was useful for confirming the trajectory to the sella and locating the anatomic midline, especially for patients requiring reoperation, in whom the landmarks had been destroyed, and for the patient with poor pneumatization of the sphenoid si-



Fig. 3 Working display and magnified view in a patient with pituitary adenoma. A: Triorthogonal reconstruction showing the position of the probe tip. B: Magnified intraoperative view. The arrow indicates the probe tip on the floor of the sella. C: Threedimensional composite color graphics of magnetic resonance and computed tomography images showing the tumor in blue, and the bilateral internal carotid arteries in red.
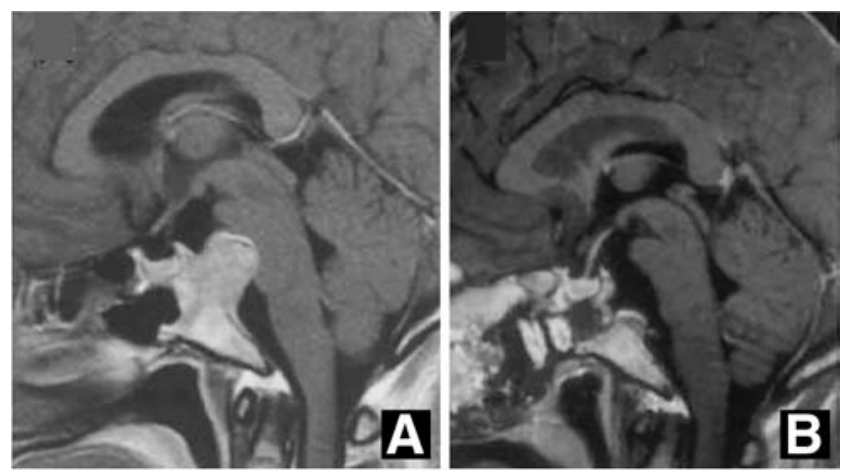

Fig. 4 Preoperative (A) and postoperative (B) magnetic resonance images in a patient with clival chordoma.

nus.

Figure 4A shows a preoperative MR image in a patient with clival chordoma. The patient underwent transsphenoidal removal of the tumor under guidance with the present system. No osteolytic change could be recognized on the clival bone. We could accurately determine the location of resection of the clival bone using the navigational system (Fig. 5A). After resection of the clival bone, we could easily and safely remove the tumor. Figure $5 \mathrm{~B}$ is a three-dimensional CT image showing the location of the clival bone resection. Postoperative MR imaging 


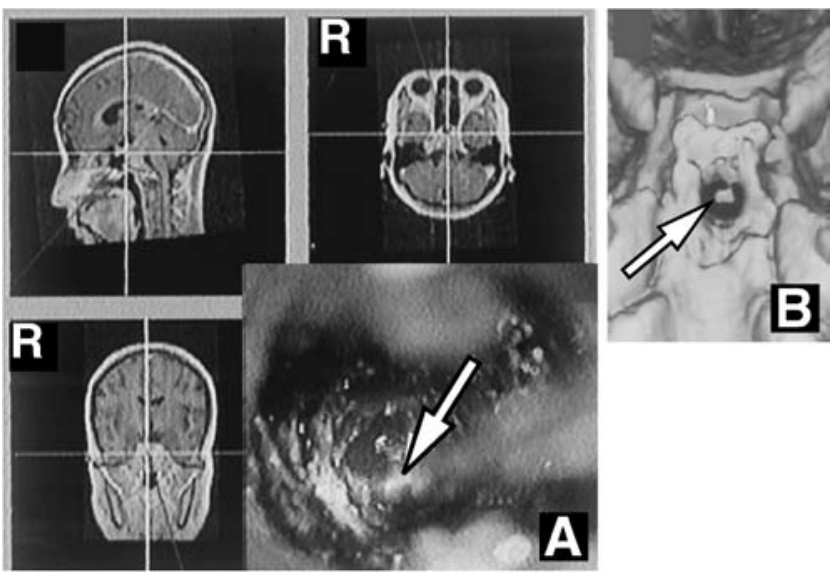

Fig. 5 The same patient as in Fig. 4. A: Triorthogonal reconstruction showing the position of the probe tip and magnified intraoperative view. The arrow indicates the probe tip. B: Three-dimensional computed tomography image showing the location of clival bone resection (arrow).

revealed total removal of the tumor (Fig. 4B).

\section{Discussion}

The articulated arm of our navigation system has an interchangeable bayonet probe, which can be used in deep structures through narrow openings, and a counterbalancing weight system so that the surgeon can hold and easily handle the probe. ${ }^{7)}$ Our navigation system could be conveniently used in skull base procedures without interfering with surgical procedures or limiting the operative field. The easy handling and unhindered microscope view permitted by our system are comparable to those achieved by an armless system and a microscope-based system. Most mechanical arm-based systems have accuracies of 1.5 to $3 \mathrm{~mm}$. ${ }^{4}$ The technical precision of our system was $1.4 \mathrm{~mm}$, and was sufficiently accurate for intraoperative localization in our series of 85 cases. The sources of error in the use of mechanical arm-based systems are attributable to the registration process and to movement of the patient's head relative to the navigational system during the craniotomy, even when the mechanical arm is affixed to the operating table or the Mayfield clamp.4) Using percutaneous bone screws or approximation of multiple randomized scalp surface points instead of fiducials can reduce the error during the registration process. ${ }^{9)}$ The reference points should be reregistered and the error corrected, if movement of the patient's head occurs relative to the navigational system or accuracy is reduced during the operation.
Recently, intraoperative guidance systems have been employed for transsphenoidal surgery. Such systems can provide continuous three-dimensional information on localization and surgical trajectory and obviate the need for intraoperative fluoroscopy. The intraoperative guidance system can be used for transsphenoidal surgery with minimal additional cost and time requirements. ${ }^{3)}$ Surgical landmarks are often difficult to determine in patients requiring reoperation or with poor pneumatization of the sphenoid sinus. However, we could safely confirm the appropriate trajectory to the sella and locate the anatomic midline using our system even in such cases. Continuous three-dimensional information on localization during intraoperative dissection provides constant awareness of the position of the surgical instrument and its relationship to the vital extrasellar structures when tumors extend laterally or superiorly beyond the sella.

Our system produces integrated three-dimensional MR and CT images, allowing simulation of the surgical procedures and intraoperative navigation using composite CGs of the scalp, brain, skull, and lesions. Preoperative assessment of the images obtained with our system aided in comprehension of the skull base anatomy and helped to select the optimal approaches. Intraoperative navigation helped to determine the actual spatial position in the skull base, the trajectory to the tumor, and the proximity of important anatomical structures. Image-guided surgery with three-dimensional composite CGs of MR and CT images effectively supported the surgeon in planning and performing skull base surgery, and may thus improve the quality and reduce the risks of complex skull base surgery.

\section{References}

1) Brinker T, Arango G, Kaminsky J, Samii A, Thorns U, Vorkapic P, Samii M: An experimental approach to image guided skull base surgery employing a microscope-based neuronavigation system. Acta Neurochir (Wien) 140: 883-889, 1998

2) Carney AS, Patel N, Baldwin DL, Coakham HB, Sandeman DR: Intra-operative image guidance in otolaryngology: the use of the ISG viewing wand. J Laryngol Otol 110: 322-327, 1996

3) Elias WJ, Chadduck JB, Alden TD, Laws ER Jr: Frameless stereotaxy for transsphenoidal surgery. Neurosurgery 45: 271-277, 1999

4) Golfinos JG, Fitzpatrick BC, Smith LR, Spetzler RF: Clinical use of a frameless stereotactic arm: results of 325 cases. J Neurosurg 83: 197-205, 1995

5) Gumprecht HK, Widenka DC, Lumenta CB: BrainLab Vector Vision Neuronavigation System: technology and clinical experiences in 131 cases. Neurosurgery 
44: 97-105, 1999

6) Hassfeld S, Zoller J, Albert FK, Wirtz CR, Knauth M, Muhling J: Preoperative planning and intraoperative navigation in skull base surgery. J Craniomaxillofac Surg 26: 220-225, 1998

7) Hayashi N, Endo S, Ikeda H, Takaku A: Neuronavigation using an articulated arm with a bayonet probe on a computer graphic composite of magnetic resonance and computerized tomography images. Minim Invasive Neurosurg 41: 144-148, 1998

8) Pillay PK: Image-guided stereotactic neurosurgery with the multicoordinate manipulator microscope. Surg Neurol 47: 171-177, 1997

9) Spetzger U, Loborde G, Glisbach JM: Frameless neuronavigation in modern neurosurgery. Minim Invasive Neurosurg 38: 163-166, 1995

Address reprint requests to: N. Hayashi, M.D., Department of Neurosurgery, Toyama Medical and Pharmaceutical University, 2630 Sugitani, Toyama 930-0194, Japan.

\section{Commentary}

The report is rich in general statements such as "safe resection of lesion in the anatomically complex area of the skull base..." What does such a statement really mean? The authors claim that they have developed a neurosurgical navigation system using three-dimensional composite computer graphics (CGs) obtained from magnetic resonance (MR) and computed tomography (CT) images, and they also claim that "these three-dimensional composite CGs of the scalp, brain, skull, and lesions allow preoperative simulation of the surgical procedures and intraoperative navigation." I do not like to comment on the neuronavigation in their 85 patients on whom the system was used during open brain surgery. However, the navigation used during surgery in 14 patients with skull base lesions is a completely different issue. In transsphenoidal surgery, in those with neuro-navigation, it is possible to get the orientation regarding the depth - and regarding the pneumatized bony compartment, the ICA location on either side as well as the bony prominences ACPs, PCPs, and other structures - visualized on the images of the MR and/or CT. A completely different issue is when one is operating on the lesions in the parasellar region in cases where the lesions are engulfing the cranial nerves. In such cases - where no imaging technique can visualize the course of individual cranial nerves through the lesion - navigation cannot help in preserving these structures. The navigation only has its value in orientating how far surgeon is from the ICA or from the bony structures. However, according to my experience with skull base surgery, this is not the issue. The issue is how to preserve the cranial nerves. It is strange (or understandable!) that throughout the report there is not a single statement regarding the cranial nerves. The authors are talking only about "safe resection." The authors concluded their discussion by saying that: "Image-guided surgery with three-dimensional composite CGs of MR and CT images effectively supported the surgeon in planning and performing skull base surgery, and may thus improve the quality and reduce the risks of complex skull base surgery." The real explanation is missing - i.e. how may the quality be improved? Objective analytical data are missing. Once again, I have to emphasize that navigation has its value in small intra-axial lesions; but by contrast, in extra-axial lesions its value is much, much less, and so far cannot be a substitute for knowledge of the anatomy, and in particular not for pathological anatomy of the cranial nerves.

Vinko V. DOLENC, M.D. Department of Neurosurgery University Hospital Center Ljubljana, Slovenia 